\title{
JOINT U.S.-EUROPEAN FUTURE COMMUNICATIONS OPERATING CONCEPT
}

\author{
John Gonda, Patricia Chávez, and Brian Hung \\ The MITRE Corporation, McLean, VA \\ Gregg Anderson, Federal Aviation Administration, Washington, D.C.
}

\begin{abstract}
The growth in aviation traffic has resulted in enormous pressure on the ability of existing spectrum resources to satisfy expected air/ground communication requirements.

To address this issue, EUROCONTROL and the Federal Aviation Administration (FAA) initiated a joint activity under the International Civil Aviation Organization (ICAO) known as the Future Communication Study (FCS) [1]. The associated work plan identifies three parts: developing communications operating concepts and requirements; conducting a technology assessment, and developing a transition and implementation roadmap.

This paper focuses on the development of initial concepts and requirements, which is one of the first steps in the process of defining a Future Radio System (FRS), a subject which should be of interest to planners in the avionics industry.
\end{abstract}

\section{Background}

The team of operational and technical experts that the FAA formed to develop the Communications Operating Concept and Requirements (COCR) for the FCS included several senior staff from MITRE/CAASD and FAA contractor personnel [2]. The associated work performed by this FCS Requirements Team was divided into the following areas: communications service identification, operational scenario development, operational safety and security assessment, communications performance requirements development, communications channel loading analysis, and application to real world traffic environments. All concepts and requirements were developed independent of any particular communications architecture for the FRS, whether of a single system or a system of systems. In addition to the primary objective of developing a communications concept and requirements that were coordinated with EUROCONTROL, the COCR was also used as an input to the FCS technology assessment and roadmap activities.

This paper highlights portions of the communications operating concept and requirements work that was accomplished by the authors.

\section{COCR Process}

To determine the overall context for future communications, the FCS Requirements Team reviewed numerous Concepts of Operation, Vision Statements, and Plans being developed and circulated by Air Navigation Service Providers (ANSPs) around the world. Armed with this understanding of various ANSPs' visions of the future of ATM communications, the following seven-step process was adopted to produce the overall COCR for the FRS:

1. The first step was to develop a notional vision and universal operating concepts for air traffic management.

2. Identification and definition of Air Traffic Services and Aeronautical Operational Control services that would be necessary to achieve the vision comprised the second step.

3. The operating environment, in which these services would be provided, was then defined to ensure all implications of each service were addressed.

4. Step four consisted of safety and security assessments for the air traffic services.

5. Using the output of 4, the high-level requirements each service would have to meet 
(so that the specified outcome or benefit of the service could be achieved safely and efficiently) were established and those requirements were allocated to the Future Radio System. Where known, existing performance requirements were used [3].

6. Next, the voice and data capacity the FRS would require in order to deliver the services was calculated.

7. By examining a few sample applications of the previous results, the seventh and final step put the COCR effort into perspective and facilitated future use.

\section{Global Air Traffic Management Concept [4]}

In general, the evolving global communications concept is that voice communications will continue to be the principal means at the start of the timeframe with a gradual shift to data communications-based operations, leaving voice communications available to perform unplanned or urgent exchanges. More automation will be introduced to enable users to transition towards a strategic and predictable Air Traffic Management (ATM) environment. Greater integration between the aircraft systems and the ground automation will allow the airspace user to fly a more efficient flight profile through negotiation based on four dimensional (4D)trajectory exchange. This will require a greater information flow between the aircraft and the ground.

\section{FCS Communications Operating Concepts and Scenarios}

The FCS is generally set in the timeframe from today through 2030.

In Phase 1 (through 2020), the roles and responsibilities of the ATM stakeholders were seen evolving from controlling to managing traffic. The paradigm change from "management by intervention" to "management by planning and intervention by exception" develops in the future ATM environment. Use of voice communication is gradually replaced by data communication services for routine and complex information transfer.
In Phase 2 (through 2030), the ATM system evolves constantly toward trajectory-based operations. All ATM stakeholders fully participate in a Layered Planning Process and the use of Collaborative Decision Making (CDM) is common place. The primary mode of communication becomes data exchange. In Phase 2, the organization of the airspace evolves into either Managed or Unmanaged categories.

Aeronautical Operational Control (AOC) communications concepts were derived from industry documents describing the services and operations supported. The AOC concept was assumed to remain constant throughout the timeframe studied.

This paper summarizes some of the team's activities and analysis conducted to understand the ATS and AOC Services that are expected to be available or desirable during the conceptual evolution to the FRS in some region within ICAO in this timeframe.

\section{Communications Services Supporting the Concepts}

Examining the preliminary operational concepts resulted in a wide range of related ATS and AOC communication services. All service domains are considered, including Airport (APT), Terminal Maneuvering Area (TMA), En route (ENR), Oceanic/Remote/Polar (ORP), and a new domain where aircraft would be delegated selfseparation known as an Autonomous Operations Area (AOA).

In Phase 1 (through approximately 2020) air traffic (ATS) and aeronautical operational control (AOC) services were developed primarily to replicate those traditionally conducted over voice radios. The following broad categories of services were identified: pilot-controller instructions/advisories, flight information, emergency, and broadcast surveillance, for example, Automatic Dependent SurveillanceBroadcast (ADS-B), Traffic Information ServicesBroadcast (TIS-B), and company-related transfers of information.

In Phase 2 (through approximately 2030) services evolve to support a much smaller set of 
very specialized operations. These include Data Link Alert (D-ALERT) Common Trajectory Coordination (COTRAC), Flight Path Intent (FLIPINT) Auto Execution (AUTOEXEC) Air-toAir Separation (AIRSEP) and Paired Approach (PAIRAPP). Supporting these services involves knowledge of the other aircraft wake vortex footprint provided by the Wake Vortex (WAKE) service. These Phase 2 services cannot be conducted through voice communications and all involve a high degree of automation to function which, in one case, AUTOEXEC - involves execution by the system without human intervention. Existing operating paradigms would obviously have to change in Phase 2 .

\section{Traffic Modeling to Support the FCS Concept}

To achieve a better understanding of the traffic density; the requirements team applied independent modeling techniques at Eurocontrol and in the U. S. using the concept phases to frame the results. The results for the U. S. National Airspace System (NAS) were derived from an analysis of all existing en route control sectors using 2004 FAA benchmark demand as applied within the MITRECAASD Mid Level Model (MLM) [5]. A 2030 Peak Instantaneous Aircraft Count (PIAC) distribution was derived which approximated Joint Planning and Development Office (JPDO) Next Generation Air Transportation System (NGATS) traffic expectations [6]. The airspace where PIAC occurred was identified as high density (HD) to allow for the most stressful loading on the communications system to be determined and used in the follow-on loading analysis.

The benchmark traffic was grown across NAS en route sectors using existing Terminal Area Forecast (TAF) based demand scenarios. U. S. modellers performed runs of existing MLM scenarios for 2004, 2013, 2020 and then used regression analysis to obtain a 2030 PIAC distribution. The 2020 and 2030 distributions developed are shown in Figures 1 and 2.

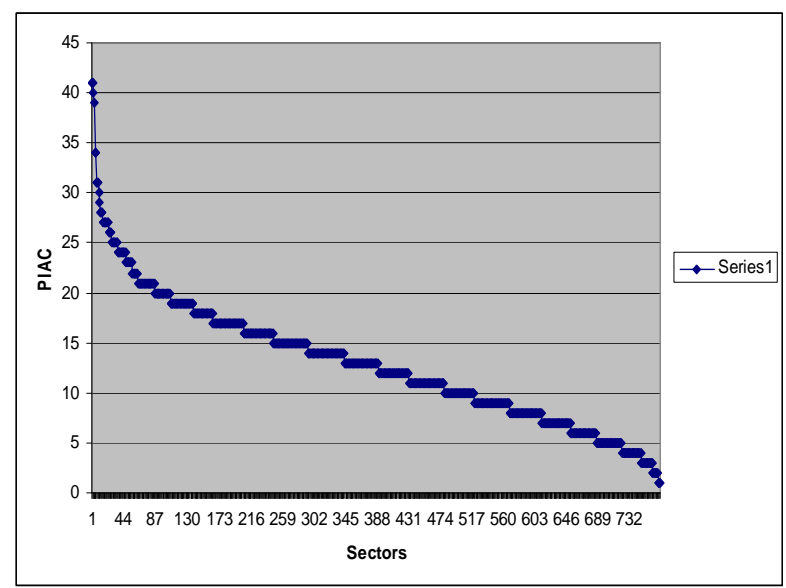

Figure 1. Phase 1 NAS En Route Sector 2020 PIAC Distribution

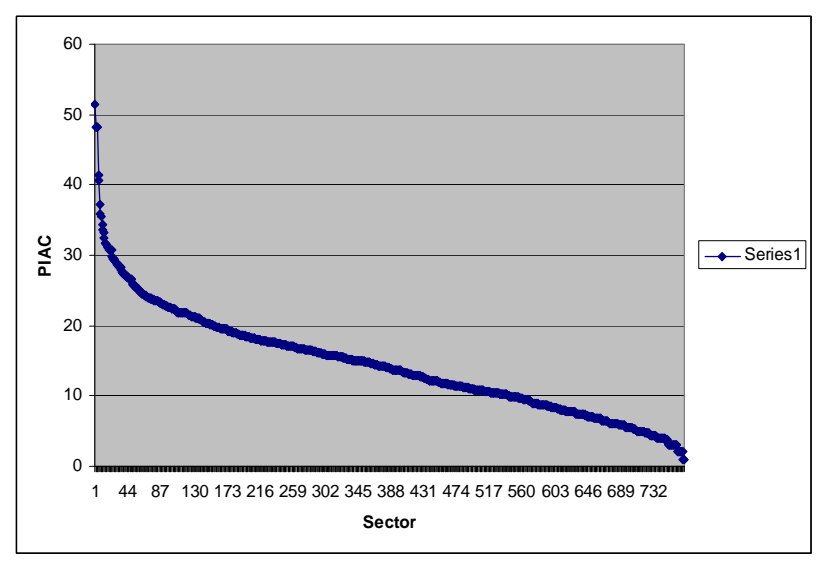

Figure 2. Phase 2 NAS En Route Sector 2030 PIAC Distribution

For the U. S. NAS density calculation, the Phase 1 High Density (HD) sector and Phase 2 HD sector were identified by picking the sector with the highest PIAC from Figures 1 and 2. Next, we identified the lower and upper altitude floors of each and then used an FAA tool that supplied the horizontal coordinates. From this information, the volume of each HD sector was calculated.

To obtain the density of each of the sectors; the PIAC for the appropriate phase was divided by the volume. Results are contained in Tables 1 and 2 below. 
Table 1. Phase 1 NAS En Route Sector Density Calculation

\begin{tabular}{|l|c|c|c|}
\hline Sector Name & PIAC & $\begin{array}{l}\text { Volume } \\
\left.\text { (nm }^{\mathbf{3}}\right)\end{array}$ & $\begin{array}{l}\text { Aircraft } \\
\text { per } \mathbf{~ m}^{\mathbf{3}}\end{array}$ \\
\hline $\begin{array}{l}\text { En route HD } \\
\text { (ZTL 019) }\end{array}$ & 41 & 7300 & 0.0056 \\
\hline
\end{tabular}

The COCR Team developed an aggregate PIAC for a three times larger sector (super sector) in 2030, to be representative of the airspace operating concept. Direct interpretation of Figure 2 and underlying data showed a maximum sector PIAC of 52 aircraft in the Atlanta Center sector ZTL019. This was identified as the NAS en route HD sector for 2030. Adjacent sectors to the en route HD sector were chosen from those closest horizontally and vertically. The result was ZTL 016 and 020 . These three sectors are actual arrival en route sectors feeding the Atlanta Hartsfield Airport. Aggregating these three sectors resulted in an example of a sector three times the size of today's sectors. The following formulas were used to aggregate the three sectors.

ZTL016 PIAC + ZTL 19 PIAC + ZTL 20

PIAC $=$ HD Super Sector PIAC

ZTL016 volume + ZTL019 volume + ZTL020 volume $=$ HD Super Sector volume

An aggregate PIAC of 95, volume of 31,996 $\mathrm{nm}^{3}$, and a resulting density shown in Table 2 were calculated for a 2030 HD Super Sector.

Table 2. Phase 2 NAS En Route Sector Density Calculation

\begin{tabular}{|l|c|c|c|}
\hline $\begin{array}{l}\text { Sector } \\
\text { Name }\end{array}$ & PIAC & $\begin{array}{l}\text { Volume } \\
(\mathbf{n m 3})\end{array}$ & $\begin{array}{l}\text { Aircraft per } \\
\mathbf{n m 3}\end{array}$ \\
\hline $\begin{array}{l}\text { En route } \\
\text { (ZTL 16) }\end{array}$ & 22 & 9816 & 0.0022 \\
\hline $\begin{array}{l}\text { En route } \\
\text { HD } \\
\text { (ZTL 19) }\end{array}$ & 52 & 7300 & 0.0071 \\
\hline $\begin{array}{l}\text { En route } \\
\text { (ZTL 20) }\end{array}$ & 21 & 14880 & 0.0014 \\
\hline $\begin{array}{l}\text { Super } \\
\text { Sector }\end{array}$ & 95 & 31996 & .0029 \\
\hline
\end{tabular}

The selected HD Super Sector is highlighted on a map of NAS en route sectors in Figure 3.

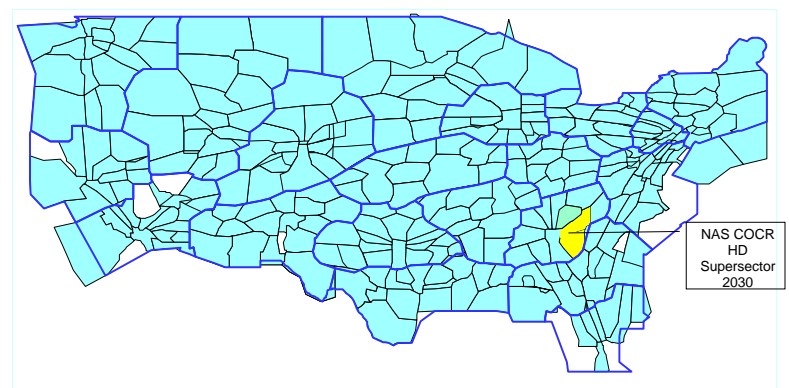

Figure 3. NAS 2005 En Route Sectors with 2030 HD En Route Super Sector Highlighted

Although other large area volumes could be represented in this fashion, these were used in this analysis. The results of the traffic modeling were used in the communications channel loading analysis which follows.

\section{Channel Loading Analysis for a Future Radio System}

A channel loading analysis was performed to estimate capacity requirements for ATS and AOC communication. In order to organize the analysis, the classes of service described in the performance requirements work were grouped into aggregate information flows. Loading for the three separate types of traffic below was performed.

- Air/Ground Voice

- Air/Ground Data

- Air/Air Data

Many of the loading input assumptions were common for all three types of analyses. For example, the PIAC in a service volume was used in both the voice and air/ground data loading analyses. The analysis presents the assumptions, the methodology used, the resultant capacity requirement, and an analysis of the results.

\section{Air/Ground Voice Loading Analysis}

The objective of the voice loading analysis was to provide estimated seconds per hour of active talk time for the party line service in each of the service volumes. Additional information regarding the 
number of transmissions per hour was also provided. Only ATS controller/pilot voice communication was analyzed as there was insufficient information to characterize AOC voice communications. The voice loading associated with broadcast channels such as for existing services known as Automatic Terminal Information Service (ATIS) is assumed to be $100 \%$.

The analysis assumed one controller position per service volume (SV) for all SVs except for the APT SV, where it can be comprised of multiple types of voice positions, i.e., ramp/clearance, ground, and tower.

The estimated seconds per hour of active pushto-talk (PTT) time and the instance information was developed using the following steps:

- A survey of existing voice studies was conducted to determine the average number of transmissions per aircraft per service volume and the average duration of each transmission [7]. One of the voice studies was an analysis of en route domain Air Traffic Control (ATC) voice transcripts that included the average number of controller and pilot transmissions per aircraft per service volume and the average duration of each transmission [8].

- The total number of seconds of voice per hour per service volume was calculated using the metrics from the voice study survey in tandem with PIAC, flight times per service volume, data link equipage rates, and voice/data utilization rates. The loading analysis used an average flight time for volumes that specify both arrival and departure aircraft durations.

- The total number of seconds of voice per hour per position was calculated from the number of positions per service volume. In addition, the occupancy and number of transmissions per hour per position was calculated.

More details of the methodology and assumptions can be found in the COCR.
Figure 4 provides the estimated voice channel occupancies derived from seconds of voice per hour per position for ATS voice communication in Phase 1 and Phase 2 for APT, TMA, ENR, and ORP SVs.

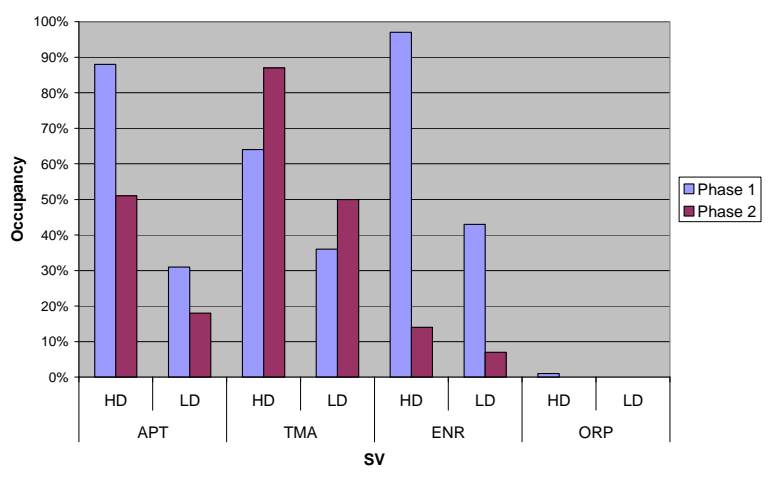

Figure 4. Voice Channel Occupancies - Phase 1 and Phase 2

For Phase 1, the estimated occupancy numbers are large compared to typical occupancy rates for some domains. This difference can be primarily attributed to the use of PIAC data instead of average aircraft counts (or aircraft per hour figures). However, some of the occupancy numbers measured at some of the busy APT, TMA, and ENR domains have been found to be close to the estimated PIAC-based occupancy numbers.

For Phase 2, the data link equipage and utilization rates are key factors that will allow the PIAC to grow while still allowing the voice channel to support the service volume. The increases in voice channel occupancies in the TMA HD and LD SVs between Phase 1 and Phase 2 can be attributed to the large increases in PIACs in those SVs in Phase 2.

\section{Air/Ground Data Loading Analysis}

The air/ground data capacity analysis examined ATS and AOC communication requirements in separate aggregate flows and within a shared aggregate flow. The aggregate flows did not include ADS-B related or air-to-air related services such as paired approach and air-to-air separation. In addition, the technology-independent data capacity analysis did not consider air/ground subnetwork overheads such as media access schemes and physical layer overhead. 
A non-pre-emptive priority queuing model was used to develop the capacity requirements in the COCR. Full details of the model and the methodology to employ it can be found in Appendix 3 of the COCR.

In the non-pre-emptive model, messages in higher priority queues (e.g. classes of service) are serviced before messages in lower priority queues. Once the server in the model has begun to transmit a message from any particular priority queue, it continues to transmit the message even if a higher priority message should arrive during transmission.

A pre-emptive resume priority queuing model was developed since publication of the COCR to provide different estimates of the capacity requirements.

In the pre-emptive resume model, once the server in the model has begun to transmit a message from any particular priority queue, the message may be interrupted by a higher priority message. The interrupted message resumes transmission after the higher priority message has completed transmission. A paper presents the non-pre-emptive and pre-emptive resume priority queuing analyses methods and the results of the analyses [9].

Figures 5, 6, 7, and 8 provide the Phase 1; Phase 2 APT and AOA SVs; Phase 2 TMA, ENR, and ORP SVs with A-EXEC service; and Phase 2 TMA, ENR, and ORP SVs without A-EXEC service estimated capacity requirements for preemptive (P) and non-pre-emptive (NP) priority queuing for the ATS and AOC traffic on the same system, assuming a single queue is used for both uplink (UL) and downlink (DL) message transmissions. To obtain upper bounds, all aircraft are assumed to be data link equipped in each domain. For each case, capacity requirements in kilobits per second (kbps) for combined uplink and downlink traffic are displayed. Results are shown for high density (HD) and low density (LD) APT, TMA, ENR, ORP, and, for Phase 2, AOA SVs.

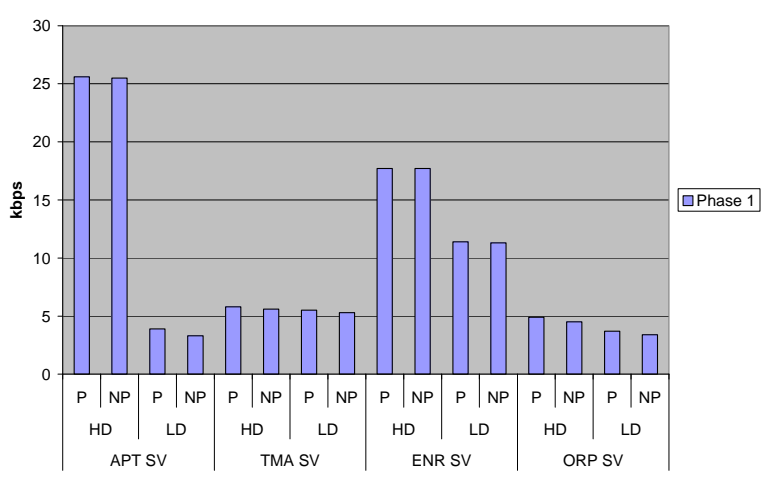

Figure 5. Air/Ground Capacity Requirements (kbps) - Phase 1

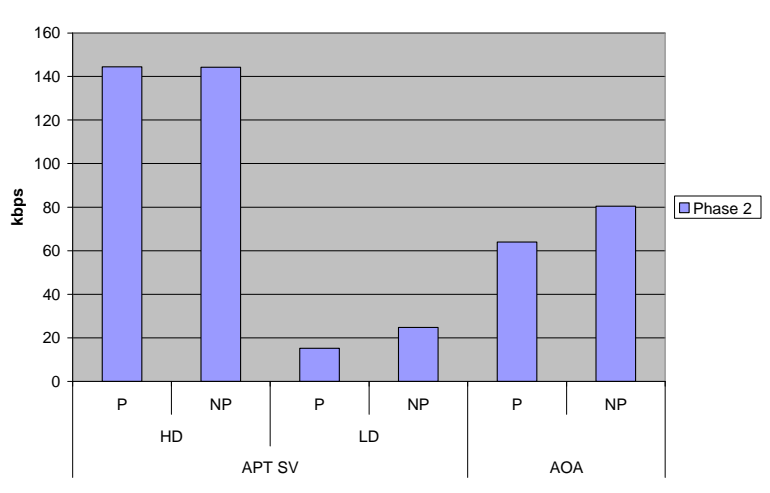

Figure 6. Air/Ground Capacity Requirements for APT and AOA SVs (kbps) - Phase 2

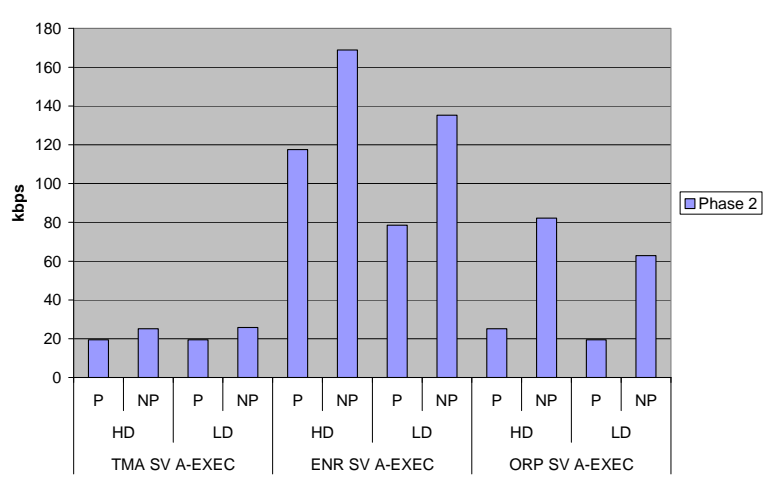

Figure 7. Air/Ground Capacity Requirements for TMA, ENR, and ORP SVs with A-EXEC (kbps) - Phase 2 


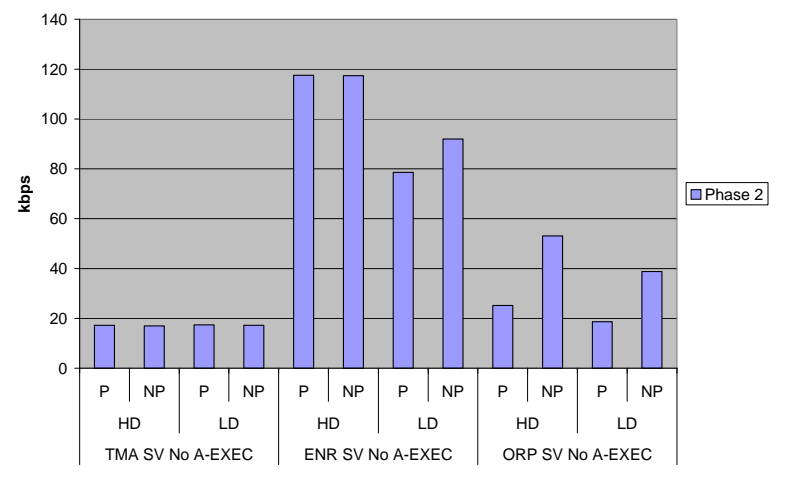

Figure 8. Air/Ground Capacity Requirements for TMA, ENR, and ORP SVs without A-EXEC (kbps) - Phase 2

The results showed that in some cases preemptive priority queuing required slightly higher capacities compared with non-pre-emptive priority queuing because lower priority messages in preemptive priority queuing tend to experience longer delays compared with non-pre-emptive priority queuing. These longer delays may exceed the 95\% delay requirements for the lower priority messages. Therefore, to meet these requirements, the required pre-emptive priority queuing capacities need to be higher. However, if there is a high-priority service requiring low 95\% delay such as the A-EXEC service, the pre-emptive priority queuing capacity requirements may be lower (in some cases much lower) than the non-pre-emptive priority queuing capacity requirements as shown in Figures 7 and 8. This is because pre-emptive priority queuing can result in much lower delays for the A-EXEC service compared with non-pre-emptive priority queuing and lower the capacity requirements.

The A-EXEC service can be a performance driver. Figures 7 and 8 show that if the A-EXEC service is included in the services the required capacity can be higher than if the A-EXEC service is not included. This is especially true for non-preemptive priority queuing.

\section{Air/Air Data Loading Analysis}

The air/air data loading analysis looked at broadcast communication services (ADS-B, TIS-B, and WAKE) and addressed air/air communication services such as PAIRAPP and AIRSEP. Both the broadcast and the addressed services were evaluated together in a single aggregate flow. Instead of SVs, the analysis used transmission volumes that corresponded to air/air ranges for each domain. The PIAC values used corresponded to the transmission volume and were different than those used for the other analyses.

The objective of the loading analysis was to estimate the required information transfer rate that must be supported by the FRS. As such, it did not consider the impacts of transmission collisions (common with 'unorganized' broadcast technology) or media access delays or scheduling overhead (common with 'organized' shared-media access technologies).

The following sections provide a brief description of the methodology and capacity requirements.

The estimated information transfer rate was developed using the following steps:

- The transmission volume PIACs and service message sizes were estimated and the FRS transmission latency was calculated using an allocated percentage of the end-to-end (E2E) latency.

- The estimated information transfer rate for each air/air service type was calculated by multiplying the PIAC times the message size and dividing the result by the FRS latency requirement.

- The information transfer rate of the aggregate flow was computed by summing the results of each air/air service type.

Details of the assumptions can be found in the COCR.

Figure 9 provides the estimated capacity requirements for air/air communication in Phase 1 and Phase 2, respectively, for ADS-B/WAKE, PAIRAPP, and AIRSEP services, assuming a single 'channel' is used for message transmissions. The analysis looked at potential information transfer rates and thus did not include the impacts of transmission collisions, media access delays and/or scheduling/message overhead. 


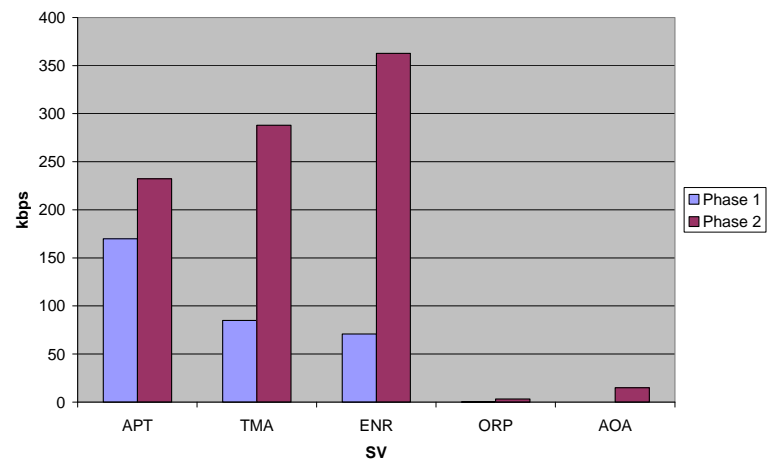

Figure 9. Air/Air Capacity Requirements (kbps) - Phase 1 and Phase 2

\section{FRS Requirements}

Safety and security requirements have an impact on the overall communications performance. To the maximum extent possible, these were considered on an end-to-end basis in compiling the COCR. The relevant requirements were then allocated to the FRS. The performance requirements that determine whether a system or service is considered safe typically include the determination of the availability, integrity, and transaction times necessary to enable safe use of the service. These performance parameters are essential ingredients that often drive system selection or design.

\section{Safety Requirements}

The primary effect of the safety assessment was on parameters such as availability and integrity in the Phase 2 timeframe. In an environment where separation standards are reduced, ground and airborne systems must have the capability to detect conflicts, provide resolutions, and in rare cases implement the resolutions (e.g., auto execution of the required maneuver by the aircraft, without human intervention). This places considerable demands on the communications systems.

Safety requirements are extremely sensitive to the service definitions. Subtle changes in a service description can significantly alter safety assessment outcomes. Much of the safety assessment process relies upon subject matter expert judgment. As such, a full, complete and common understanding of a service and environment is required before safety-derived performance requirements can be verified and validated.

\section{Security Requirements}

Misuse of information exchanged between ANSPs, aircraft, and users can have serious safety, efficiency and financial impact. Since keeping sensitive information out of the wrong hands and preventing information from being misused is especially critical to aviation, security analysis is an important source of FRS requirements. How sensitive the information is, what risks or threats are posed and how likely they are, and what damage could be inflicted are compiled to characterize security objectives. Security requirements to be imposed on the communications system were derived from these objectives.

Key Findings of the Information Security analysis include [10]:

- The security requirements were undertaken on an end-to-end basis and therefore many of the security requirements are beyond outside the scope of the FRS. One security requirement that is directly relevant to the FRS is the need for some level of protection against deliberate radio frequency (RF) interference.

- The FRS should have the ability to use message security features, such as message authentication, as needed to ensure safe delivery of services that require high integrity messaging.

\section{Performance Requirements}

The FRS technology selection, system design, and implementation will, to a great degree, be driven by the performance requirements allocated to the FRS. Most significant of these performance requirements are availability, integrity, and latency. In addition, as information security attributes are both difficult and expensive to implement as augmentations to a system, the ability of a technology to provide information security must also be addressed. For each data service, the severity of the worst-case operational hazard was determined under two cases: a data integrity failure 
and a loss of service failure. These two assessments generated the Service Level Operational Hazard Severity classifications. In addition, the safety and business interest impact of the information associated with each service being intercepted, redirected, or replaced was evaluated to determine the Service Level Confidentiality classification. Finally, the largest latency (message delay) that could be tolerated during delivery of a service was derived.

Since the Service Level Operational Hazard and Confidentiality classifications and the Service Latencies are determined by examining the conduct and delivery of the service, they establish how strong the whole chain of components in the service delivery thread has to be. The FRS is but one link in the chain, which would include automation, other ground systems and networks, other airborne systems and networks, etc. So, the next step was to decide what portions of these Service Level requirements would have to be met by the FRS. Allocated latency, integrity and availability requirements were derived. Finally, classes of communications services were established to group together services that shared similar allocated performance requirements.

Performance requirements for Voice services were developed with a more direct approach. Since the Operating Concept envisioned the voice function would support the same types of services presently offered (but perhaps in lesser quantities), the performance requirements (i.e., call establishment, latency, availability, and confidentiality) were derived from existing voice system requirements documents.

The most stringent FRS-allocated data requirements are highlighted in Table 11 below.

Table 11. Most Stringent FRS-Allocated Data Requirements

\begin{tabular}{|l|l|l|c|c|c|c|c|c|c|}
\hline \multirow{2}{*}{\begin{tabular}{l} 
Service \\
\multirow{2}{*}{$\begin{array}{l}\text { Phase } \\
\text { Pha }\end{array}$}
\end{tabular}} & \multirow{2}{*}{$\begin{array}{l}\text { Service } \\
\text { Type }\end{array}$} & Confidentiality & APT & TMA & ENR & ORP & AOA & $\begin{array}{l}\text { Integrity } \\
\text { FRS }\end{array}$ & $\begin{array}{l}\text { Availability } \\
\text { Of } \\
\text { Provision } \\
\text { FRS }\end{array}$ \\
\hline $\begin{array}{l}\text { ATS } \\
\text { Phase 1 }\end{array}$ & Broadcast & Medium & 0.8 & 4.8 & 9.6 & 9.6 & - & $5 \mathrm{E}-06$ & 0.9965 \\
\hline & Addressed & Medium & 3.8 & 3.8 & 3.8 & 26.5 & - & $5 \mathrm{E}-06$ & 0.9965 \\
\hline $\begin{array}{l}\text { ATS } \\
\text { Phase 2 }\end{array}$ & Broadcast & Medium & 0.8 & 2.4 & 2.4 & 2.4 & 2.4 & $1 \mathrm{E}-06$ & $1-(5.0 \mathrm{E}-6)$ \\
\hline & Addressed & Medium & 1.4 & 0.74 & 0.74 & 0.74 & 1.4 & $5 \mathrm{E}-10$ & $1-(5.0 \mathrm{E}-8)$ \\
\hline $\begin{array}{l}\text { AOC } \\
1+2\end{array}$ & - & Medium & 13.60 & 13.60 & 13.60 & 26.50 & 26.5 & $5.0 \mathrm{E}-8$ & $1-(5.0 \mathrm{E}-5)$ \\
\hline
\end{tabular}

ATS services that generate stringent requirements, which might merit further consideration, include the following:

- $\quad$ D-ALERT drives ATS Phase 1 confidentiality

- A-EXEC drives ATS Phase 2 integrity and availability
- $\quad$ PAIRAPP and A-EXEC drive ATS Phase 2 latency

- $\quad$ ADS-B and TIS-B drive broadcast performance requirements

The FRS voice requirements identified are shown in Table 12 below. 
Table 12. Voice Performance Requirements (ATS) - Phase 1 \& 2

\begin{tabular}{|l|l|l|l|l|l|}
\hline Service Type & \multicolumn{4}{|c|}{ Party-Line } & Broadcast \\
\hline Domain & APT HD & TMA HD & ENR HD & ORP HD & ALL \\
\hline $\begin{array}{l}\text { Call } \\
\text { Establishment } \\
\text { Delay }\end{array}$ & $50 \mathrm{~ms}$ & $50 \mathrm{~ms}$ & $50 \mathrm{~ms}$ & $200 \mathrm{~ms}$ & $20 \mathrm{~s}$ \\
\hline Voice Latency & $250 \mathrm{~ms}$ & $250 \mathrm{~ms}$ & $250 \mathrm{~ms}$ & $485 \mathrm{~ms}$ & $485 \mathrm{~ms}$ \\
\hline $\mathrm{A}_{\mathrm{P}}$ & 0.99999 & 0.99999 & 0.99999 & 0.99999 & 0.999 \\
\hline $\mathrm{A}_{\mathrm{U}}$ & 0.99998 & 0.99998 & 0.99998 & 0.99998 & 0.998 \\
\hline
\end{tabular}

\section{Summary}

Communications are a critical component of air traffic management. Existing voice and data communications are becoming a systemic bottleneck, constraining capacity, security, and safety improvements. Various proposals have been offered to address these communications-based constraints, but none has achieved global endorsement. The Communications Operating Concept and Requirements (COCR) has three primary goals:

- to develop international understanding of air traffic management concepts and services which the future aviation communications system should support, based on future strategy documents

- to document fundamental Future Radio System requirements to facilitate subsequent FCS technology assessments

- to support the ANSPs' iterative process of operational service selection, by identifying the full range of services on which to begin safety, cost/benefit, or other analyses.

The results appear to indicate that capacity requirements for future ATS air/ground data are relatively modest, especially when compared to the high capacities promised by future technologies. However, this problem has more than one dimension, as delivery of air traffic services requires simultaneous achievement of many, often challenging, requirements. This safety-driven combination of capacity, integrity, reliability, latency and coverage requirements have typically dictated unique solutions for aviation. Current system capabilities must be assessed to determine under what conditions Phase 1 requirements could be met, or if enhancements would be necessary. While Phase 2 requirements appear to be beyond the capabilities of systems currently deployed, numerous advanced technologies, as well as options for further evolution of today's most capable systems, should meet all but the most demanding needs. To implement this two-phased vision affordably, careful examination of the services, especially those that drive requirements, will be necessary to balance costs and benefits. In the meantime, the FCS Requirements Team will update and expand on these requirements in COCR version 2, to be released in mid 2007.

\section{References}

[1] Aeronautical Communications Panel (ACP) Working Group C, 19-23 April 2004, Future Communications Study, $7^{\text {th }}$ meeting, Montreal, Canada.

[2] Federal Aviation Administration, ATO-P, May 2006, Communications Operating Concept and Requirements, Version 1, Washington, D.C.

[3] RTCA/EUROCAE, 29 April 2004, Safety and Performance Requirements Standard for Air Traffic Data Link Services in Continental Airspace RTCA DO-290/EUROCAE ED-120, Washington, D.C.

[4] ICAO, 2003, Global ATM Operational Concept for CNS/ATM, 11th Air Navigation Conference, International Civil Aviation Organization, Montreal, Canada.

[5] MITRE-CAASD, 2005, Mid Level Model User's Handbook, McLean, VA 
[6] US Department of Transportation, December 2004, Next Generation Air Transportation System (NGATS) Integrated Plan.

[7] DeVito, Katherine, 7 March 2005, "A Voice Study Survey: Characterizing Voice Channel Access by Airspace Domain", COCR-PSG-KD-14, Washington, D.C.

[8] Hung, B, April 2005, “MITRE-Sponsored Research: An Analysis of En Route Domain Air Traffic Control Voice Transcripts”, McLean, VA.
[9] Hung, B, 1-3 May 2006, A Method for Estimating Air/Ground Data Capacity Requirements, 2006 NASA ICNS Conference and Workshop, Baltimore, MD.

[10] EUROCONTROL/FAA, September 2005, Security Analysis Supporting the Communications Operating Concept and Requirements for the Future Radio System, Washington, D.C.

$25^{\text {th }}$ Digital Avionics Systems Conference October 15, 2006 